\title{
POSTER
}

\section{Radiothérapie oto-rhino-laryngée et altérations des capacités gustatives}

\section{Desoutter $A^{1,2}$, Pommier $P^{3}$, Salino $S^{2}$, Bodard AG ${ }^{1,2}$}

1 - Service de Consultations et Traitements Dentaires, Hospices Civils de Lyon, 6 place Depéret, 69007 Lyon et UFR d'odontologie, 11 rue Guillaume Paradin, 69008 Lyon

2 - Centre Léon Bérard, odontologie, 28 rue Laennec, Lyon

3 - Centre Léon Bérard, radiothérapie, 28 rue Laennec, Lyon

\section{Introduction}

La radiothérapie est une modalité thérapeutique quasiment systématique dans la prise en charge des cancers oto-rhino-laryngés (ORL). Elle présente toutefois de nombreux effets indésirables à court terme (mucite...) et à long terme (risque d'ostéoradionécrose, hyposialie...).

À travers son rôle dans l'hyposialie et dans la modification des papilles gustatives, la radiothérapie va engendrer des perturbations quantitatives et qualitatives du goût (hypogueusie et dysgueusie). Ces troubles surviennent en cours de traitement, et persistent plusieurs semaines, voire plusieurs mois ou années après la fin de la radiothérapie. Ils entraînent des difficultés pour l'alimentation, pouvant mettre en péril le statut nutritionnel du patient, et impactent fortement la qualité de vie.

L'objectif de cette étude est d'évaluer les caractéristiques de l'altération du goût chez les patients ayant bénéficié d'une radiothérapie de la sphère ORL.

Matériels et méthodes

Une revue de la littérature sur Pubmed a été réalisée, en utilisant les mots clés " taste», " radiotherapy ", " head and neck neoplasms ", " taste/radiation effects ». Les critères de sélection des articles étaient : langue anglaise ou française, résumé disponible sur Pubmed. Les revues de la littérature et les reports de cas ont été exclus.

\section{Résultats}

Les troubles du goût chez les patients irradiés pour un cancer ORL sont connus depuis de nombreuses années (1). Les différentes études réalisées sur l'altération du goût pendant une radiothérapie ORL s'accordent sur le fait que les troubles gustatifs apparaissent dès la deuxième semaine de traitement et touchent les quatre saveurs (2). Ces troubles sont plus marqués si la partie antérieure de la langue est inclue dans les champs d'irradiation $(2,3)$. Toutefois, le degré de sévérité et les caractéristiques de ces modifications sont controversés. Par exemple, pour certains, la capacité à détecter la saveur sucrée serait diminuée $(2,3)$. Pour d'autres, la saveur sucrée serait mieux préservée que les saveurs amère et salée, car de nombreux bourgeons du goût lui seraient dédiés (1). Enfin, il existe un désaccord sur l'évolution dans le temps de ces troubles du goût. Selon certaines études, un recouvrement des capacités gustatives serait possible 2 à 6 mois après la fin de la radiothérapie $(2,3)$. Pour d'autres, il n'y aurait pas de relation entre le degré d'atteinte et le temps écoulé après la fin de la radiothérapie (1). 
Discussion et conclusion

La radiothérapie de la sphère ORL entraîne des modifications du goût qui touchent les quatre saveurs. Ces modifications surviennent en cours de radiothérapie (dès la deuxième semaine de traitement en général), et se poursuivent à l'arrêt du traitement, sans qu'un consensus ne soit établi quant au caractère réversible de ces troubles. Par ailleurs, l'aspect hédonique des perturbations gustatives n'a pas été exploré à notre connaissance.

\section{Références}

1. Mossman K et al. Int J Radiat Oncol Biol Phys. 1982; 8(6):991-7.

2. Kamprad F et al. Strahlenther Onkol Organ Dtsch Röntgenges Al. 2008; 184(3):157-62.

3. Yamashita H et al. Int J Radiat Oncol Biol Phys. 2006; 66(5):1422-9. 